\title{
Tunable Polymer Fiber Bragg Grating (FBG) Inscription: Fabrication of Dual-FBG Temperature Compensated Polymer Optical Fiber Strain Sensors
}

\author{
Yuan, Scott Wu; Stefani, Alessio; Bang, Ole
}

Published in:

I E E E Photonics Technology Letters

Link to article, DOI:

10.1109/LPT.2011.2179927

Publication date:

2012

Document Version

Peer reviewed version

Link back to DTU Orbit

Citation (APA):

Yuan, S. W., Stefani, A., \& Bang, O. (2012). Tunable Polymer Fiber Bragg Grating (FBG) Inscription: Fabrication of Dual-FBG Temperature Compensated Polymer Optical Fiber Strain Sensors. I E E E Photonics Technology Letters, 24(5), 401-403. https://doi.org/10.1109/LPT.2011.2179927

\section{General rights}

Copyright and moral rights for the publications made accessible in the public portal are retained by the authors and/or other copyright owners and it is a condition of accessing publications that users recognise and abide by the legal requirements associated with these rights.

- Users may download and print one copy of any publication from the public portal for the purpose of private study or research.

- You may not further distribute the material or use it for any profit-making activity or commercial gain

- You may freely distribute the URL identifying the publication in the public portal 


\title{
Tunable polymer Fiber Bragg Grating (FBG) inscription: Fabrication of dual-FBG temperature compensated polymer optical fiber strain sensors
}

\author{
Wu Yuan, Alessio Stefani, Ole Bang* \\ DTU Fotonik, Department of Photonics Engineering, Technical University of Denmark, DK-2800 Kongens Lyngby, Denmark \\ *Corresponding author: oban@fotonik.dtu.dk
}

\begin{abstract}
We demonstrate stable wavelength tunable inscription of polymer optical fiber Bragg gratings (FBGs). By straining the fiber during FBG inscription we linearly tune the center wavelength over $7 \mathrm{~nm}$ with less than $1 \%$ strain. Above $1 \%$ strain the tuning curve saturates and we show a maximum tuning of $12 \mathrm{~nm}$ with $2.25 \%$ strain. We use this inscription method to fabricate a dual-FBG strain sensor in a poly (methyl methacrylate) (PMMA) single-mode microstructured polymer optical fiber and demonstrate temperature compensated strain sensing around $850 \mathrm{~nm}$.
\end{abstract}

Due to the low Young's modulus (about 25 times lower than silica) and high elastic limit of over 10\% (about 10 times higher than silica), fiber Bragg gratings (FBGs) in polymer optical fibers (POFs) are attractive for fiberoptical strain sensing [1-2]. POFs are also clinically acceptable, flexible and non-brittle, which makes the POF FBG a candidate for in-vivo biomedical applications [3-6]. FBGs have been reported in both step index POFs [2,7-9] and microstructured POFs (mPOFs) [9-13].

To date the majority of POFs and mPOFs are made of PMMA, which has a high thermo-optic coefficient and strongly absorbs water. PMMA FBG strain sensors therefore have a large cross-sensitivity to humidity and temperature $[1,8,13]$. The problem of humidity is strongly reduced by using POF FBGs made of the polymer TOPAS $[4,5]$, which has a humidity sensitivity of less than 38.4 $\mathrm{pm} / \% \mathrm{rH} @ 1565 \mathrm{~nm}$ [13]. This is more than 50 times less than POF FBGs made of PMMA [10,13]. However, both TOPAS and PMMA POF FBGs are still sensitive to temperature with similar sensitivities $[1,13]$. This is a major problem for POF (and silica) FBG strain sensors in practical applications, in particular in static strain sensing, where temperature variations occur on the same time-scale as the variations in strain.

A simple solution is to use a second closely spaced and strain free FBG with a different resonance wavelength to provide an independent control of the temperature. The difference in resonance frequency between the two FBGs will then still measure the strain, but be independent of temperature, as was demonstrated for silica FBGs [14].

In this paper we demonstrate the concept of dual-FBG temperature compensated strain sensing for POF FBGs. To fabricate two (or more) POF FBGs with closely spaced resonance wavelengths we further demonstrate a simple technique for highly controlled tuning of the resonance wavelength of a POF FBG written with the standard phase-mask technique using the same phase-mask. By straining the POF during writing we can linearly tune the wavelength by $7 \mathrm{~nm}$ using only $1 \%$ strain. Going into the saturated regime we show $12 \mathrm{~nm}$ tuning with $2.25 \%$ strain using a force of only $0.5 \mathrm{~N}$ due to the low Young's modulus of PMMA. This tuning range is about 5 times higher than for silica fibers [15] and can prove useful for future multiplexed sensor applications of POFs.

In an earlier experiment, two broadband FBGs were inscribed in a large-core multi-mode $\mathrm{mPOF}$ at $1562 \mathrm{~nm}$ and $1545 \mathrm{~nm}$ using also a single phase-mask, by thermally annealing the first grating before writing the next [16]. Our method differs from the annealing technique, in that it is much more controllable and works with POFs regardless of their drawing conditions and whether they have been annealed or not. Furthermore, we are here able to apply it to narrow-band single-mode POFs to demonstrate closely packed FBGs ideal for future multiplexed POF FBG strain sensors. Most importantly, we use the technique to present the first demonstration of temperature compensated FBG strain sensing in POFs.

In our experiments we use an endlessly single-mode PMMA mPOF fabricated by the drill-and-draw technique. The mPOF has a diameter of $180 \mu \mathrm{m}$ and a solid core surrounded by three rings of air holes arranged in a hexagonal lattice. The air-hole diameter is on average $2 \pm 0.2 \mu \mathrm{m}$ and the inter-hole pitch is on average $4.8 \pm 0.2$ $\mu \mathrm{m}$ (see inset of Fig. 1(a)). The hole diameter to pitch ratio is thus $\mathrm{d} / \Lambda \approx 0.41$, which is below the threshold value of 0.42 that ensures endlessly single-mode operation [9].

We use a $325 \mathrm{~nm}$ HeCd CW laser (IK5751I-G, Kimmon) and a phase-mask with a uniform period of $572.4 \mathrm{~nm}$ (Ibsen Photonics) customized for $325 \mathrm{~nm}$ writing of $\mathrm{mPOF}$ FBGs with a resonance wavelength of $\lambda_{B}=850 \mathrm{~nm}$. The fiber was supported by v-grooves on both sides of a gap to avoid reflection, and strain was applied to control the wavelength and keep the fiber straight during writing. Two hours of exposure time was used and the resulting gratings have a length of $10 \mathrm{~mm}$. The applied strain was measured with a v-groove axial force sensor (FSC102, Thorlabs) and the FBG reflection spectrum was measured in reflection with a spectral resolution of $10 \mathrm{pm}$ using an $850 \mathrm{~nm}$ silica fiber circulator, a SuperK Versa broadband source (NKT Photonics), and an optical spectrum analyzer (Ando AQ6317B). A standard singlemode silica fiber was butt-coupled to the $\mathrm{mPOF}$ using an angle cleaved end-facet and a small amount of refractive index matching gel in order to reduce Fresnel reflections. 
In Fig. 1(a) we show that $\lambda_{\mathrm{B}}$ depends linearly on the applied force and strain for strains up to $1 \%$, with a total tunability of $7 \mathrm{~nm}$. In this regime $\lambda_{\mathrm{B}}$ relaxes rapidly to its stationary value, after which it remains stable, as seen in Fig. 1(b). For strains above $1 \%$ the tuning curve saturates and the relaxation time increases significantly. This is a reflection of the visco-elastic properties of the PMMA, which become particularly apparent when the fiber is exposed to high strain for long times [1].
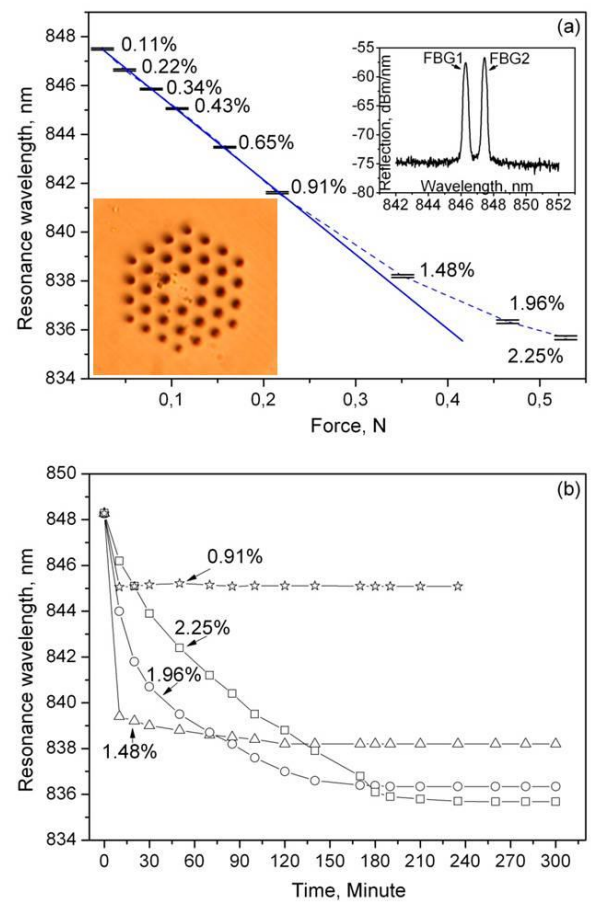

Fig. 1. (a) Resulting FBG resonance wavelength versus force. The corresponding strain is given at the measurement points. Inset shows the $\mathrm{mPOF}$ used for grating writing and the reflection spectrum of a fabricated dual-FBG; (b) Relaxation of the resonance wavelength (848.3 $\mathrm{nm}$ while strained) after release for different applied strains.

In the inset of Fig. 1(a) we show two FBGs with $\lambda_{\mathrm{B}}=$ $846.28 \mathrm{~nm}$ (FBG1) and $847.44 \mathrm{~nm}$ (FBG2) inscribed in the same fiber with $1 \mathrm{~cm}$ separation. Both FBGs were unchanged after storage for more than 24 hours. We now use this dual-FBG as a strain sensor to demonstrate temperature compensated strain sensing. We control the temperature of both gratings and mechanically stretch FBG2, while FBG1 remains unstretched. The two ends of FBG2 are glued to micro-translation stages with a UV curable glue (OG116+31, Epotek), which is mechanically much stiffer than the PMMA mPOF, so that it does not influence the strain. One stage was kept fixed, while the other moved to apply axial strain to FBG2 manually with a low loading speed. The axial strain was determined by dividing the fiber longitudinal elongation by the length of fiber between the two gluing points. The longitudinal displacement accuracy of the translation stage is 0.01 $\mathrm{mm}$. The strain-free FBG1, was taped on the fixed stage. Another micro-translation stage was used to butt-couple the mPOF to a single-mode silica fiber (SM800, Thorlabs). The FBGs were heated up with a resistive heater (TH60, Linkam) placed on top of them. A thermocouple was used to measure the temperature as close to the gratings as possible with an uncertainty around $0.3^{\circ} \mathrm{C}$. A humidity sensor (C210, Lufft) was used to monitor the humidity near the two gratings with an uncertainty of $0.5 \% \mathrm{rH}$.

The gratings were heated up from room temperature to $36.3^{\circ} \mathrm{C}$ stepwise in a single cycle with the temperature allowed to stabilize for twenty minutes at each new set temperature before the reflection spectra were measured. The relative humidity decreased correspondingly from 66 $\% \mathrm{rH}$ to $44 \% \mathrm{rH}$. In Fig. 2(a) we show the measured dependence of the resonance wavelength of FBG1 and FBG2 on the temperature, with the corresponding humidity indicated on the curve. A nearly identical blue shift of both resonance wavelengths is observed, while there was no obvious change in the bandwidth (not shown). A temperature sensitivity of $-77 \pm 7 \mathrm{pm} /{ }^{\circ} \mathrm{C}$ was found for both gratings by a linear fit, which corresponds well with earlier measurements [1].
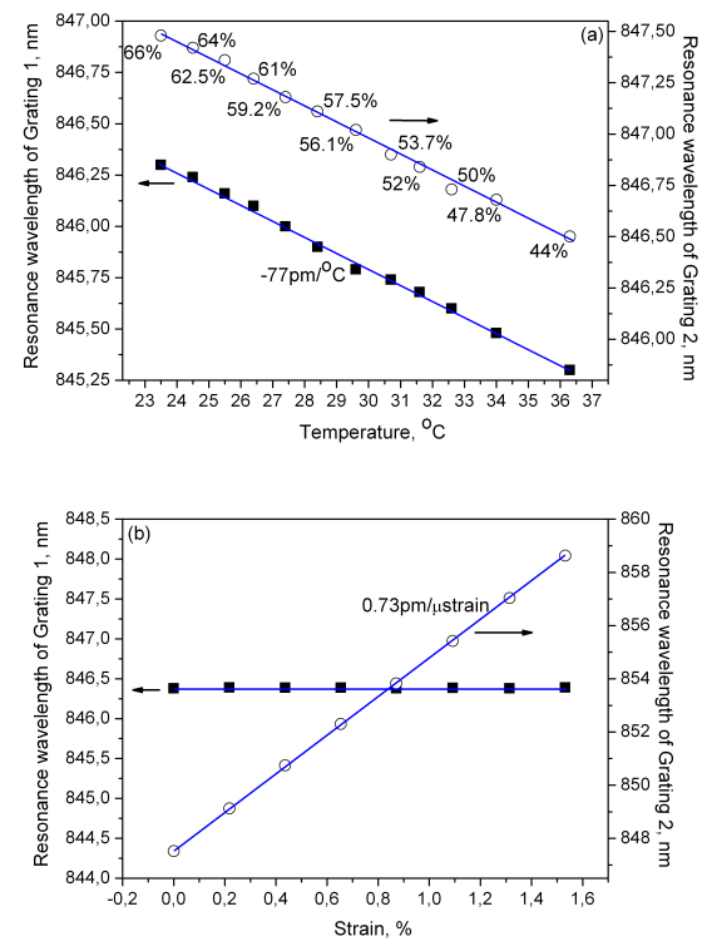

Fig. 2. Resonance wavelength of FBG 1 and 2 (a) versus temperature/humidity with no strain and (b) versus strain applied to only FBG 2 at a fixed temperature (humidity) of $25^{\circ} \mathrm{C}(63 \%)$.

A strain loading experiment of FBG2 was carried out with a fix temperature of $25^{\circ} \mathrm{C}$ (constant humidity of $63 \% \mathrm{rH}$ ). The grating was left to stabilize for about ten minutes each time the tensile strain was changed before reading the reflection spectrum. As seen in Fig. 2(b), FBG2 shows a linear response of the center wavelength over the whole strain loading range up to $1.53 \%$ strain, 
with a linear fit giving a sensitivity of $0.73 \pm 0.02$ $\mathrm{pm} / \mathrm{\mu strain}$ as also found in [9]. The resonance wavelength of the unstrained FBG1 is stable as expected.
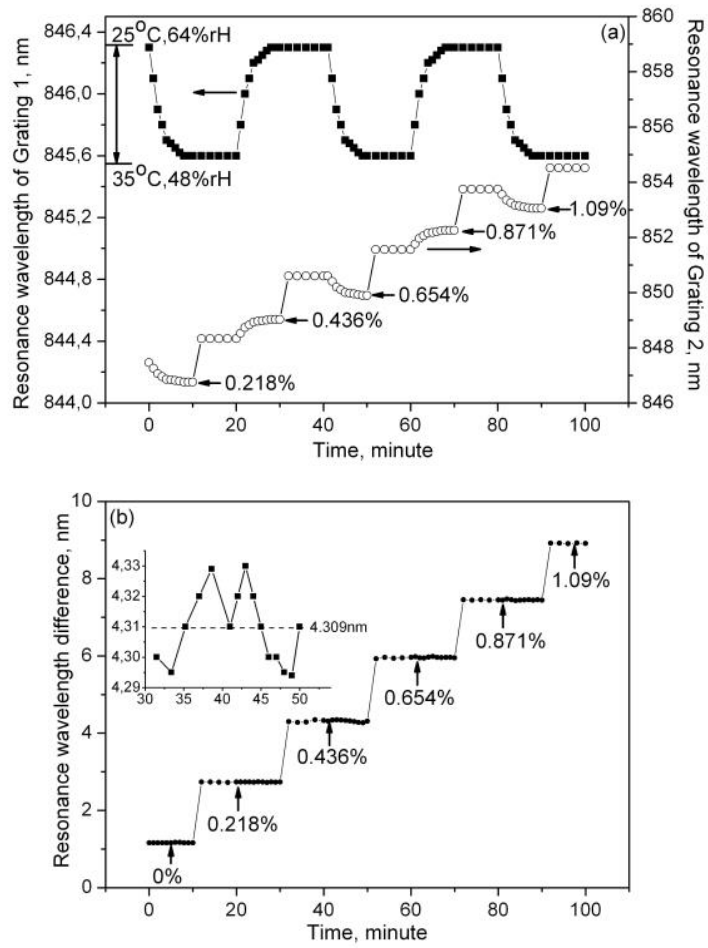

Fig. 3. (a) Resonance wavelength of FBG1 and FBG2 subject to a temperature cycle alternating between 25 and $35{ }^{\circ} \mathrm{C}$ every $20 \mathrm{~min}$. (corresponding humidity 64 and $38 \% \mathrm{rH}$ ) while the strain applied to FBG2 only was increased every 20 min. starting after 10 min. (b) Difference in resonance wavelength of FBG2 and FBG1, showing temperature compensation. Inset shows a zoom

In order to emulate a practical situation and further confirm the temperature (humidity) compensation capability of our dual-FBG strain sensor, a strain-sensing experiment with a periodic temperature (humidity) change was carried out. As shown in Fig. 3 (a), strain was gradually applied to FBG2 every $20 \mathrm{~min}$., while the temperature (humidity) experienced by both gratings was cycled between $25^{\circ} \mathrm{C}(64 \% \mathrm{rH})$ and $35^{\circ} \mathrm{C}(48 \% \mathrm{rH})$ every 20 $\mathrm{min}$. with a $10 \mathrm{~min}$. offset from the strain increase. In Fig. 3(a) we see how the strain recorded by FBG2 is strongly affected by the changing temperature (humidity), leading to the expected slow change in center wavelength of $77 \mathrm{pm}$ with every $10^{\circ} \mathrm{C}$ change. The temperature dependence is effectively compensated by instead monitoring the difference in center wavelength between FBG2 and FBG1, as demonstrated in Fig. 3(b). In fact one cannot talk about a cross-sensitivity to temperature of this sensor, because the fluctuations observed in the zoom in Fig. 3(b) are so small that they are within the limits of the mechanical stability of the set-up. If we instead analyse the plateaus or periods with fixed strain, then the maximum standard deviation from the mean of $12 \mathrm{pm}$ is found for the third plateau from 30 to $50 \mathrm{~min}$. shown in the zoom. If we then divide the $12 \mathrm{pm}$ with the change in average wavelength of $1687 \mathrm{pm}$ going to the plateau 3 from plateau 2 , then we get a measure for the maximum noise of $0.71 \%$. This is obviously a very low degree of noise given that the temperature is increased suddenly by $10^{\circ} \mathrm{C}$ during this $20 \mathrm{~min}$. period.

In summary, we have demonstrated a temperature and humidity compensated POF FBG strain sensor, which is based on a dual-FBG architecture in a single-mode PMMA mPOF and operates at the low-loss wavelength $850 \mathrm{~nm}$. We have also demonstrated a simple technique to UV-write POF FBGs with a highly controlled tunable resonance wavelength, still using only a single phasemask. Applying strain to the POF during writing we linearly tune the resonance by $7 \mathrm{~nm}$ using only $1 \%$ strain and show that going into the saturation regime allows to tune the FBG resonance by $12 \mathrm{~nm}$ with $2.25 \%$ strain.

The strain tuning technique has been applied to silica fibers before but with a limited tunability of only $2.5 \mathrm{~nm}$ due to the high Young's modulus of silica. The $12 \mathrm{~nm}$ we demonstrated here is thus by far the highest ever achieved with this technique.

\section{References}

1. D.J.Webb and K. Kalli: 'Polymer fiber Bragg gratings', in Fiber Bragg Grating Sensors: Recent Advancements, Industrial Applications and Market Exploitation, A. Cusano, A. Cutolo, J. Albert (Eds.), ch. 15, pp. 1-20. (Bentham Science Publishers Ltd. 2009)

2. W. Yuan, A. Stefani, M. Bache, T. Jacobsen, B. Rose, N. Herholdt-Rasmussen, F.K. Nielsen, S. Andresen, O.B. Sørensen, K.S. Hansen, O. Bang, "Improved thermal and strain performance of annealed polymer optical fiber Bragg gratings," Opt. Commun. 284, 176-182 (2010).

3. J. Jensen, P. Hoiby, G. Emiliyanov, O. Bang, L, Pedersen, A. Bjarklev, "Selective detection of antibodies in microstructured polymer optical fibers," Opt. Express 13, 5883-5889 (2005).

4. G. Emiliyanov, J.B. Jensen, O. Bang, P.E. Hoiby, L.H. Pedersen, E.M. Kjær, L. Lindvold, "Localized biosensing with TOPAS microstructured polymer optical fiber," Opt. Lett. 32, 460-462 (2007).

5. G. Emiliyanov, J.B. Jensen, O. Bang, P.E. Hoiby, L.H Pedersen, E.M. Kjaer, L. Lindvold, "Localized biosensing with TOPAS microstructured polymer optical fiber: Erratum," Opt. Lett. 32, 1059 (2007).

6. C. Markos, W. Yuan, K. Vlachos, G.E. Town, O. Bang, "Label-free biosensing with high sensitivity in dual-core microstructured polymer optical fibers," Opt. Express 19, 7790-7798 (2011).

7. Z. Xiong, G.D. Peng, B. Wu, P.L. Chu, "Highly tunable Bragg gratings in single-mode polymer optical fibers", IEEE Photon. Technol. Lett. 11, 352-354 (1999).

8. C. Zhang, W. Zhang, D.J. Webb, G.D. Peng, "Optical fibre temperature and humidity sensor," Electron. Lett. 46, 643-644 (2010).

9. A. Stefani, W. Yuan, C. Markos, O. Bang, "Narrow bandwidth $850 \mathrm{~nm}$ fiber Bragg gratings in few-mode polymer optical fibers," IEEE Photon. Technol. Lett. 23, 660-662 (2011).

10. H. Dobb, D.J. Webb, K. Kalli, A. Argyros, M.C.J. Large, M.A. van Eijkelenborg "Continous wave ultraviolet light-induced fiber Bragg gratings in few and single mode microstructured polymer optical fibers", Opt. Lett. 30, 3296-3298 (2005). 
11. I.P. Johnson, K. Kalli, D.J. Webb, " $827 \mathrm{~nm}$ Bragg grating sensor in multimode microstructured polymer optical fiber," Electron. Lett. 46, 1217-1218 (2010).

12. I.P. Johnson, W. Yuan, A. Stefani, K.Nielsen, H.K

Rasmussen, L. Khan, D.J. Webb, K. Kalli, O. Bang, "Optical fibre Bragg grating recorded in TOPAS cyclic olefin copolymer", Electron. Lett. 47, 271-272 (2011).

13. W. Yuan, L. Khan, D. J. Webb, K. Kalli, H. K. Rasmussen, A. Stefani, O. Bang, "Humidity insensitive TOPAS polymer fiber Bragg grating sensor,” Opt. Express 19, 19731-19739 (2011).

14. F. M. Haran, J.K. Rew, P.D. Foote, “A strain-isolated fibre bragg grating sensor for temperature compensation of fibre Bragg grating strain sensors,” Meas. Sci. Technol. 9, 11631166 (1998)

15. Q. Zhang, D.A. Brown, L. Reinhart, T.F. Morse, J.Q. Wang, G. Xiao, "Tuning Bragg wavelength by writing gratings on prestrained fibers”, IEEE Photon. Technol. Lett. 6, 839 (1994).

16. I.P. Johnson, D.J. Webb, K. Kalli, M.C.J. Large, A. Argyros, "Multiplexed FBG sensor recorded in multimode microstructured polymer optical fibre", Proc. SPIE 7714, 77140D (2010); doi:10.1117/12.854410 